\title{
Herzstück: Performatives Lehren und Lernen am Beispiel eines Gemeinschaftsprojekts im universitären DaF-Bereich oder frei nach Schiller: Von der performativen Erziehung des Menschen
}

\author{
Nina Kulovics, Kerstin Terler
}

\begin{abstract}
Dieser Artikel berichtet von einem Gemeinschaftsprojekt unter ÖsterreichlektorInnen aus dem Wintersemester 2013/14 vorwiegend am Beispiel der französischen Universitäten Bordeaux und Mulhouse. Er soll als Erfahrungsbericht und Impuls dienen, in dem Entstehung, Rahmenbedingungen, Ziele, pädagogische Vorgehensweise und Resümee vorgestellt werden.
\end{abstract}

\section{Projektvorstellung}

Im Wintersemester 2013/14 schloss sich eine Gruppe von ÖsterreichlektorInnen zu einem länderübergreifenden Gemeinschaftsprojekt zusammen, in dem das Theaterstück Herzstück des deutschen Autors Heiner Müller im Rahmen eines sechs europäische Staaten umfassenden Videowettbewerbs von jeweils einer Studentengruppe pro Standort szenisch dargestellt werden sollte. Den Grundimpuls hierfür gab der von Maik Walter geleitete Theaterworkshop Theaterpädagogik und Fremdsprachenvermittlung im Zuge des berufsbegleitenden Zertifikatskurses Kompetenzfeld Auslandslektorat ${ }^{1}$ für OeAD ${ }^{2}$-LektorInnen am Postgraduate Center der Universität Wien. Aus der Arbeitsanweisung, theaterpädagogische Übungen in unserem Fremdsprachenunterricht einzusetzen, entstanden weitere kleinere bzw. größere Gemeinschaftsprojekte, deren Ergebnisse uns vom Einsatz von Theatermethoden ${ }^{3}$ im Fremdsprachenunterricht überzeugten.

\footnotetext{
${ }^{1}$ Vgl. http://www.postgraduatecenter.at/corporate-programs/kompetenzfeldauslandslektorat (Accessed 15 September 2014).

${ }^{2}$ Österreichische Austauschdienst-GmbH: http://www.oead.at (Accessed 15 September 2014).

${ }^{3}$ Eine strenge Unterscheidung zwischen Theater- und Dramapädagogik, wie in der Sekundärliteratur nachzulesen ist, spielte in unserem Projekt eine untergeordnete Rolle.
} 
In dem hier vorgestellten Gemeinschaftsprojekt wurde in einem Zeitraum von drei Monaten in einem dramapädagogisch motivierten Annäherungsprozess an den literarischen Text ein Endprodukt, diverse Interpretationen/Wahrnehmungen, in Form von Kurzvideos inner- und außerhalb des Unterrichts zum mündlichen Ausdruck realisiert. Nachdem jeder Standort ein erstes internes Klassensiegervideo einschickte, wurden anschließend via Facebook-Gruppe und Dropbox diese Videos weitergeleitet und - ganz im Sinne eines Eurovision Video Contests - von den anderen Studentengruppen begutachtet, um schließlich ein Siegervideo aus allen teilnehmenden Ländern zu küren. Die Kriterien zum Voting wurden hierbei nicht von den Lehrenden vorgegeben, sondern erfolgten durch die Studierenden selbst. Als Gewinner ging der Beitrag der Nationalen Iwan-Franko-Universität Lemberg (Lviv), Ukraine hervor. Die Studentinnen erhielten in Anlehnung an das Stück Milkaherzen den USB-Stick „Dein Schlüssel zu Österreich“4 des ÖDaF-Verbandes. Die in der Dramapädagogik zu verortende szenische Umsetzung soll hier insbesondere anhand der Universitäten Bordeaux und Mulhouse illustriert werden.

\section{Rahmenbedingungen und Zielsetzungen}

\subsection{Rahmenbedingungen}

Die Zielgruppe umfasste Studierende der Germanistik und Angewandten Fremdsprachen im ersten Studienjahr im Rahmen von Kursen zum mündlichen Ausdruck. Die Gesamtheit der Studierenden dieses Jahrgangs wirkte bei diesem Projekt mit, wobei die überwiegende Mehrheit keinerlei Theatererfahrung besaß. Da das Sprachniveau vor allem zu Beginn des Studiums noch sehr heterogen sein kann, lag es nach den Kriterien des Gemeinsamen europäischen Referenzrahmens $(\mathrm{GeR})$ bei A2 bis C2. Als Zeitrahmen für die diversen Phasen zur Erarbeitung des Stückes wurden zwei Stunden (120 Minuten) anberaumt. Bei den zeitlichen, räumlichen und technischen Ressourcen musste auf die jeweiligen Standorte Rücksicht genommen werden.

\subsection{Zielsetzungen}

Ein übergeordnetes Ziel war es, den Studenten mittels dramapädagogischer Annäherung die Scheu vor der in der Fremdsprache geschriebenen, oft als schwierig empfundenen deutschsprachigen Literatur zu nehmen und so performativ komplementär zu der im universitären Umfeld vorrangigen kognitivhermeneutisch-interpretatorischen Begegnung mit Literatur zu wirken. Damit wurde auch der Forderung der letzten Jahre nach einer performativen Wende in der Fremdsprachendidaktik gefolgt (vgl. Hallet 2010; Schewe 2011). Darüber hinaus wird mit performativem Lehren und Lernen den GeR-Richtlinien eines

\footnotetext{
${ }^{4}$ Dieser wurde durch den OeAD zur Verfügung gestellt und war ein Geschenk der österreichischen DaF/DaZ-Institutionen an die TeilnehmerInnen der IDT (=Internationale Deutschlehrertagung) 2013 in Bozen.
} 
handlungs- und aufgabenorientierten Fremdsprachenlernens entsprochen, da Sprachlernende sich als sprachlich Handelnde in sozialen Kontexten befinden und in kommunikativen Settings agieren. ${ }^{5}$ Des Weiteren wird das soziale Element durch Teamarbeit, Interaktion, selbstständiges und aktives Handeln gefördert, was mancherorts im französischen Universitätsalltag (noch) kein (didaktisches) Thema ist. ${ }^{6}$ Medienkompetenz wird ebenso trainiert. Auch der Förderung von Motivation wird im $\mathrm{GeR}$ ihre Wichtigkeit eingeräumt. Man weiß zudem aus Neurowissenschaft und Neurolinguistik, dass möglichst positive Emotionen an der Aneignung einer fremden Sprache großen Anteil haben, da sie für die kognitive Verankerung im Gehirn sorgen und somit die Lernleistung steigern können (vgl. Even 2003; Sambanis 2013). In der Inszenierung als Widerspiegelung der Wahrnehmungswelt der unterschiedlichen Zielsprachenländer passiert im Perspektivenwechsel - vom Eigenen ins Fremde und wieder zurück - interkulturelle Erfahrung, eine weitere wichtige Kompetenz. ${ }^{7}$ Hier kann auch eine interkulturell-methodische Brücke zur französischen Simulation globale geschlagen werden, die in den 1970er Jahren unter Francis Debyser und Jean-Marc Caré vom Centre International d'Études Pédagogiques (C.I.E.P.) in Sèvres entwickelt wurde. ${ }^{8}$

\section{Pädagogischer Aufbau}

Dramapädagogik ist ein Ansatz, der die Mittel des Theaters zu pädagogischen Zwecken einsetzt. Im Vordergrund steht dabei nicht primär das Ergebnis, nämlich die Produktion eines Theaterstücks, sondern der Lernprozess in allen seinen Dimensionen: physisch, ästhetisch (sinnlich), emotional und kognitiv. (Tselikas 1999: 21)

Performative Prozesse integrieren als alternatives, interaktives, kreatives und kooperatives Lernen Kunst und andere Wissens- und Lebensbereiche des Menschen, indem Poetisches durch körperliches Handeln erlebbar gemacht wird und somit eine neue Wahrnehmung der fremden Sprache sowohl für Akteure als auch Rezipienten gefördert werden kann. In der prozess- und produktionsorientierten Auseinandersetzung mit Sprache und Literatur wird das kreativ-künstlerische Element gefördert (vgl. Oelschläger 2004: 24), ein authentischer Zugang zur Fremdsprache und ein offenes, angenehmes Lernklima angestrebt.

\footnotetext{
${ }^{5}$ Vgl. http://www.goethe.de/z/50/commeuro/201.htm (Accessed 15 September 2014).

${ }^{6}$ Stichwort: Gruppendynamik/Persönlichkeitsentwicklung, vgl. auch Even 2003: 147.

7 Näheres zu interkulturellen Erfahrungen durch Theaterarbeit, vgl. Aden 2010 und Sting 2008.

${ }^{8}$ Diese ist als handlungsorientierte, performative Unterrichtsform für alle Altersstufen geeignet, in der innerhalb eines vorgegebenen Rahmens Lerner peu à peu eine eigene Welt erfinden, in der sie „ihr“ Leben leben. Kreativität und spielerische Identifikation mit d en Rollen u nd die Lernerzentrierung sorgen für Motivation und einen authentischeren Gebrauch der Fremdsprache. Der Lehrende fungiert als Regisseur und Coach und flicht d ie n otwendigen sprachlichen Strukturen ein. Vgl. verschiedene Aufsätze von Debyser und Caré, u.a. Caré Debyser 1995.
} 


\subsection{Aufwärm- und Vorübungen}

Der Einstieg in die zweistündige Sequenz ${ }^{9}$ zur Erarbeitung des Kurztextes Herzstück von Heiner Müller erfolgte durch eine thematische Sensibilisierung mit Hilfe von Aufwärmübungen (Bewegungs-, Körper- und Stimmübungen, wie etwa Name \& Geste), die auch als Lockerungsübungen auf allen Sprachniveaus nach dem GeR durchgeführt werden können. Diese Übungen dienen der Bewusstseinswerdung, der Konzentrationssteigerung und sollen auch das Vertrauen innerhalb der Großgruppe auf- bzw. ausbauen. Abschließend wurden vorbereitende Übungen zum Herzstück aus dem Bereich der Dramapädagogik und Dramagrammatik (vgl. Tselikas 1999; Even 2003) eingesetzt. Diese Phase wurde in allen teilnehmenden Ländern in abgeänderter Form gestaltet, an den Universitäten Bordeaux und Mulhouse stand an dieser Stelle die Vorbesprechung der in- und außerhalb des Textes zu findenden Redewendungen, die das Wort Herz enthalten (ein reines Herz haben, das Herz am rechten Fleck haben, jemandem sein Herz zu Füßen legen etc.). Neben der Wortschatzerweiterung wurde auf die Aussprache, insbesondere von Frikativa und Affrikata eingegangen (Herz, verzweifeln, Ziegelstein, schmutzig, Vergnügen), was vor allem für visuelle und haptische Lerntypen von Vorteil ist. Die Redewendungen wurden anschließend mit der Arbeitsanweisung verteilt, diese nun in der Gruppe als Standbilder darzustellen. Die anderen Gruppen sollten durch Ja-Nein-Fragen und vor allem durch die inszenierte Gestik und Mimik auf die jeweils zu erratende Wendung stoßen. Die vorbereitenden Übungen können generell auf allen Niveaustufen eingesetzt werden.

\subsection{Text- und Szenenarbeit}

Der Kontakt mit dem gesamten Kurztext erfolgte u.a. durch das chorische Sprechen, um den gesamten Kurztext gemeinsam einzuüben (Texteinprägung durch rhythmisches Sprechen, Stimm- und Sprechtraining, Wegfall von Sprechhemmungen) und um dadurch die Gruppendynamik zu fördern. So soll auch in die künstlerisch-ästhetische Als-ob-Realität eingestiegen und die Spiellust geweckt werden. Es erfolgte eine Teilung in Gruppe A und B, wobei Gruppe A den ersten Satz des Stückes gemeinsam aussprach, woraufhin Gruppe B auch im Chor mit dem zweiten Satz antwortete. Dies wurde mit dem gesamten Kurztext wiederholt. Die Übung kann mehrmals wiederholt und variiert werden (traurig/glücklich, ernst/lustig, laut/leise etc.). Generell ist das chorische Sprechen auf allen Niveaustufen anwendbar. Durch das mehrmalige Repetieren prägt man sich das Theaterstück und die Aussprache in spielerischer Form besser ein. Auch für eher unsichere, schüchterne KursteilnehmerInnen ist diese Übung von Vorteil, da es sich um eine Gruppenaktivität handelt und Fehler bzw. Ausspracheunsicherheiten weniger auffallen bzw. zum Tragen kommen.

\footnotetext{
${ }^{9}$ In Anlehnung an Maik Walters Theaterworkshop 2012 und unter Einarbeitung weiterer einschlägiger Fachliteratur (Vgl. Holl 2011; Küppers 2011; Tselikas 1999; Walter 2012) konzipierten wir den Kursverlauf, adaptiert an die standortbedingten Ressourcen (Zielgruppe, Zeit, Raum, Technik).
} 
Nun erfolgte die autonome Szenenarbeit in kleineren Arbeitsgruppen von drei bis sechs Personen, die - aufgrund der Zeitvorgabe - ungefähr 30 Minuten andauern sollte.

\subsection{Präsentation - Abschluss - Nachbereitung}

Auf die Erarbeitungsphase in der Gruppe folgte die Präsentationsphase, in der die Szene vor Klasse und Kamera im Klassenraum mit den vorhandenen Ressourcen gespielt wurde. Einige besonders engagierte Studierende in Bordeaux drehten die Videos auch außerhalb des Unterrichts und untermalten diese mit Musik. Die szenische Umsetzung war von sehr unterschiedlichen Interpretationen und Inszenierungen gekennzeichnet (siehe 4.1). Als Abschluss wurde ein Blitzlicht durchgeführt, an dieser Stelle wurden Eindrücke bzw. Meinungen zum Erlebten gesammelt und somit auch Sprach- und Sprachlernreflexion a ngestrebt (Was nehme ich inhaltlich, sprachlich und persönlich aus diesen Einheiten mit?). In der folgenden Stunde wurde abermals darauf eingegangen, der Wortschatz gefestigt und sprachliche Korrekturen in Phonetik, Grammatik und Lexik vorgenommen. Zudem wurden die Videos der anderen teilnehmenden Universitäten gezeigt und bewertet. Als Preis für die Klassensiegervideos wurden auch Milkaherzen verteilt, wobei das wichtigste Kriterium zur Wahl desselbigen in Mulhouse und Bordeaux eher in der humoristischen und performativen Umsetzung der Stücke lag. Alternativ könnte man das Theaterstück kreativ umschreiben lassen, was im Zuge eines Kurses zur mündlichen Kommunikation aber nur dann sinnvoll ist, wenn der Schreibprozess als Hausaufgabe ausgelagert wird.

\section{Ergebnisse - Beobachtungen - Mehrwert - Fazit}

\subsection{Ergebnisse}

Die szenische Umsetzung des auf einem Kurzdialog basierenden Stückes brachte je nach studentischer Interpretation unterschiedliche interessante und sehenswerte Ergebnisse auf thematischer, sozialer und ästhetischer Ebene hervor. Die unterschiedlichen kulturellen „Sprachen“ (Sting 2008: 105) und ethnischen Bezüge wurden in den Videos deutlich sichtbar. Während das Siegervideo aus Lemberg über Länder und Gesellschaften hinweg den weltumfassenden Kapitalismus und seine mitunter fatalen Folgen (hier: Organhandel) sozialkritisch beäugte, wurde das zeitlose Stück in Bordeaux z.B. in Form eines Raps ${ }^{10}$ - mit (deutscher) Musik unterlegt - in die französische Gegenwart geholt (Kulturtransfer). Damit wurde im künstlerisch-ästhetischen Element mit Sprache verbal und Bewegung/Tanz nonverbal Kritik an so mancher gesellschaftlicher Oberflächlichkeit (hier: französisches TV-Sternchen Nabilla) geübt. In Mulhouse hingegen integrierte eine Studentengruppe, die

\footnotetext{
${ }^{10}$ Dieses Videobeispiel kann auf einem einschlägigen Internet-Videoportal eingesehen wer-
} den. 
wiederum verstärkt auf nonverbale Kommunikation und Körpersprache setzte, das Stück in ihre eigene Lebenswelt (hier: unerwiderte Liebe).

\subsection{Beobachtungen/Mehrwert}

Durch die körperliche Visualisierung des literarischen Textes konnte Abwechslung in den meist rein kognitiv geprägten Unterrichtsalltag gebracht werden, was von vielen Studierenden nach anfänglicher Skepsis bzw. Befangenheit durchwegs positiv bewertet wurde. Es konnte auch beobachtet werden, dass Teamarbeit, selbstständiges und aktives Handeln die soziale Interaktion in der Gruppe positiv beeinflusste. Auch der Perspektivenwechsel - vom Eigenen ins Fremde und wieder zurück - bzw. die interkulturelle Erfahrung war für die Studierenden eine Bereicherung. Zu sehen, was andere Länder bzw. Kulturen aus dem Stück herauslesen, wie inszeniert wurde, welche Themen gesetzt wurden. Sensible Ohren widmeten sich außerdem den Akzenten. Es handelte sich folglich um eine „vitale kulturelle und soziale Begegnung“ (Sting 2008: 107). Die Motivation war allgemein höher, was erfreulicherweise auch zu weniger Sprechhemmungen führte:

Der lockernde Umgang mit der theatralischen Distanzierung kann dazu beitragen, Ängste des Identitätsverlusts, des Fehlermachens, des 'DanebenSeins' zu überwinden, mit der Rolle der Deutschsprachigen zu spielen und diese sogar zu geniessen [sic!]. (Tselikas 1999: 33)

\section{Fazit}

Lehren und Lernen „mit Kopf, Herz, Hand und Fuß“ (vgl. Schewe 1993: 44ff.) fördert auf alternative, ganzheitliche, handlungsbasierte, interaktive, kreative, performative, künstlerische, prozess- und produktionsorientierte Weise die Auseinandersetzung mit Sprache und Literatur. Alle Ressourcen des Lerners (inklusive der technischen), das kognitive, physische, psychische, kreative und soziale Moment des Fremdsprachenlernens werden ausgeschöpft, Körper, Geist und Seele vereinigt und mediale Kompetenz gefördert. Der internationale Wettbewerbs- und Projektcharakter war ein zusätzlicher wichtiger Motivationsfaktor. Interessant war dieser länderübergreifende Aspekt nicht zuletzt deshalb, weil er sowohl Lernende als auch Lehrende zusätzlich motivierte, Sprache und Literatur zu erleben, zu gestalten und (um)zuformen.

\section{Schlusswort}

Frei nach dem Motto oser dépasser les frontières ${ }^{11}$ wollten wir performatives Lehren und Lernen in den französischen DaF-Unterricht integrieren bzw. aufnehmen. Nach ersten Einblicken in Theatermethoden im Rahmen diverser

${ }^{11}$ Zitiert nach: http://www. uha.fr/novatris (Accessed 15 September 2014). 
didaktischer Ausbildungen, u.a. bei Tselikas und Winklbauer, war Walters Workshop ausschlaggebend für eine erweiterte Beschäftigung und einen vermehrten Unterrichtseinsatz. Die Beiträge, Workshops und Auftritte, die im Rahmen der Konferenz Performative Teaching, Learning and Research (University College Cork, 2014) durchgeführt wurden, zeigten erneut das Potential von performativen Lehr- und Lernmethoden auf. In diesem Sinne:

Denn, um es endlich auf einmal herauszusagen, der Mensch spielt nur, wo er in voller Bedeutung des Worts Mensch ist, und er ist nur da ganz Mensch, wo er spielt. (Schiller, nach Göpfert; Fricke 1966: 481).

\section{Bibliografie}

Aden, Joëlle (2010): Theaterspielen als Chance in der interkulturellen Begegnung. Projekt ANRAT - IDEA Europe. Berlin: Schibri

Caré, Jean-Marc; Debyser, Francis (1995): Simulations Globales. Sèvres: Centre International d'Études Pédagogiques (C.I.E.P.)

Even, Susanne (2003): Drama Grammatik. Dramapädagogische Ansätze für den Grammatikunterricht Deutsch als Fremdsprache. München: iudicium

Gemeinsamer europäischer Referenzrahmen für Sprachen: Lernen, lehren, beurteilen. http://www.goethe.de/z/50/commeuro/i0.htm (Accessed 15 September 2014)

Hallet, Wolfgang (2010): Performative Kompetenz und Fremdsprachenunterricht. In: Scenario IV, 1, 5-18

Holl, Edda (2011): Sprach-Fluss. Theaterübungen für Sprachunterricht und interkulturelles Lernen. München: Hueber

Küppers, Almut et al. (Hrsg.) (2011): Inszenierungen im Fremdsprachenunterricht: Grundlagen, Formen, Perspektiven. Braunschweig: Schroedel, Diesterweg, Klinkhardt

NovaTris, Centre de compétences transfrontalières, Université Haute-Alsace:

http://www . uha.fr/novatris(Accessed 15 September 2014)

Oelschläger, Birgit (2004): Szenisches Spiel im Unterricht „Deutsch als Fremdsprache". In: GFL-Journal 1, 24-34

Österreichische Austauschdienst-GmbH: http://www. oead.at (Accessed 15 September 2014)

Postgraduate Center der Universität Wien:

http://www.postgraduatecenter.at/corporateprograms/kompetenzfeld-auslandslektorat (Accessed 15 September 2014)

Sambanis, Michaela (2013): Fremdsprachenunterricht und Neurowissenschaften. Tübingen: Narr

Schewe, Manfred (1993): Fremdsprache inszenieren: zur theoretischen Fundierung einer dramapädagogischen Lehr- und Lernpraxis. Oldenburg: Zentrum für Pädagogische Berufspraxis, Universität Oldenburg 
Schewe, Manfred (2011): Die Welt auch im fremdsprachlichen Unterricht immer wieder neu verzaubern: Plädoyer für eine performative Lehr- und Lernkultur! In: Küppers, Almut et al. (Hrsg.): Inszenierungen im Fremdsprachenunterricht: Grundlagen, Formen, Perspektiven. Kempten: Diesterweg, 20-31

Schiller, Friedrich: Über die ästhetische Erziehung des Menschen in einer Reihe von Briefen (1795). In: Göpfert, Herbert; Fricke, Gerhard (Hrsg.) (1966): Sämtliche Werke. Bd. II. München: Hanser, 445-520

Sting, Wolfgang (2008): Anderes Sehen. Interkulturelles Theater und Theaterpädagogik. In: Hoffmann, Klaus; Klose, Rainer (Hrsg.): Theater interkulturell. Theaterarbeit mit Kindern und Jugendlichen. Uckerland/Milow: Schibri, 101-109

Tselikas, Elektra I. (1999): Dramapädagogik im Sprachunterricht. Zürich: Orell Füssli

Walter, Maik (2012): Theater in der Fremdsprachenvermittlung. In: Nix, Christoph et al. (Hrsg.): Lektionen 5: Theaterpädagogik. Berlin: Theater der Zeit, 182-188 